

\title{
Real-time analysis in Run 3 with the LHCb experiment
}

\author{
Peilian $\mathbf{L i}^{1, *}$ \\ Heidelberg University, \\ Im Neuenheimer Feld 226, Heidelberg, Germany \\ E-mail: peilian.li@cern.ch
}

The second stage of LHCb experiment's high-level trigger, deployed on a CPU server farm, not only selects events but performs an offline-quality alignment and calibration of the detector and uses this information to allow physics analysts to deploy essentially their full offline analysis level selections (including computing isolation, flavour tagging, etc) at the trigger level. This "real time analysis" concept has also allowed LHCb to fully unify its online and offline software codebases. We cover the design and performance of the system which will be deployed in Run 3, with particular attention to the physics performance of the new algorithms.

*** The European Physical Society Conference on High Energy Physics (EPS-HEP2021), ***

*** 26-30 July $2021 * * *$

*** Online conference, jointly organized by Universität Hamburg and the research center DESY ***

${ }^{1}$ On behalf of the LHCb Collaboration

* Speaker 


\section{Introduction}

The LHCb experiment is dedicated to the study of $\mathrm{CP}$ violation phenomena and rare decays in the heavy flavour quark sector at the LHC with proton-proton (pp) and heavy ion collisions. Over the years 2011-2018, both the LHC machine and the LHCb detector performed extremely well, providing great improvements in the pioneering $\mathrm{CP}$ violation measurements of $\mathrm{B}$ and $\mathrm{D}$ decays [1, 2], observation of the rarest beauty meson decays [3], discovery of pentaquarks [4] and interesting hints of anomalies [5], which have stimulated widespread attention in both theory and experiment communities. However, many of these measurements are still statistics-limited, which requires the upgrade of $\mathrm{LHCb}$ detector with increased event rate and efficiency, to further pursue these exciting results and fully exploit the flavour physics potential of the LHC.

The LHCb upgrade for Run 3 is planned to start data taking in 2022 at $\sqrt{s}=14 \mathrm{TeV}^{1}$ with five-times higher instantaneous luminosity $\left(2 \times 10^{33} \mathrm{~cm}^{-2} \mathrm{~s}^{-1}\right)$ compared with Run 2 [9]. Many of the sub-detectors and all electronics systems are being replaced to enable a reliable and robust detector operating at higher luminosity without compromising the excellent physics performance of the Run 2 detector. At this luminosity, corresponding to an average of six pp collisions per LHC bunch crossing, it is no longer possible to efficiently identify bunch crossings of interest based purely on the calorimeter and muon system information, due to too much QCD background generated by the pileup [6]. The rates of beauty and charm hadrons in acceptance reach the MHz level, making it impossible to save the full information of all decays in the limited offline storage.

For these reasons, $\mathrm{LHCb}$ has decided to remove the hardware trigger and directly process the full $30 \mathrm{MHz}$ of bunch crossing data in two software-based High Level Triggers (HLTs). This allows much more sophisticated selections, in particular the use of tracking information at the very first trigger stage, and a reduced event model in the second trigger stage which allows LHCb to persist more signal candidates for the same amount of storage space. The first HLT stage, HLT1, will be deployed using GPU cards installed in the Event Builder (EB) servers. It will partially reconstruct the event with high momentum tracks passing through the whole detector, and reduce the event rate by a factor of 30 to 60, using inclusive one and two-track based selection algorithms. More details of HLT1 can be found in Ref. [7]. This proceeding focuses on the design and performance of the HLT2 system, which is deployed on a CPU server farm, with particular attention to the physics performance of the new algorithms and a brief introduction to the concept of LHCb's reduced event model, known as selective persistence.

\section{Reconstruction and physics performance}

The events filtered by HLT1 will be buffered on an array of disks for quasi-real-time detector alignment and calibration [8] to maintain the ultimate detector performance and to ensure the offlinequality reconstruction in HLT2. The task of HLT2 is to fully reconstruct offline-fidelity tracks in full momentum range and neutral objects, including track fitting [12] and particle identification. Huge efforts have been devoted to improve the performance by parallelising the algorithms and optimising the framework and event model. The throughput is defined as the number of events

\footnotetext{
${ }^{1}$ For the commissioning year, the actual beam energy might be relatively lower, e.g. $13.5 \mathrm{TeV}$, as well as the actual luminosity.
} 
which can be processed by a given architecture per second in steady-state conditions. Figure 1 shows the throughput and breakdown among algorithms for the HLT2 reconstruction (without selection and persistence yet) with the "fastest" configuration ${ }^{2}$. This throughput, about 383 events per second per node, is extrapolated from measurements using an Intel(R) Xeon(R) CPU E5-2630 v4 machine in integration tests and largely exceeds the design throughput [10] of about 250 events/s/node ${ }^{3}$.

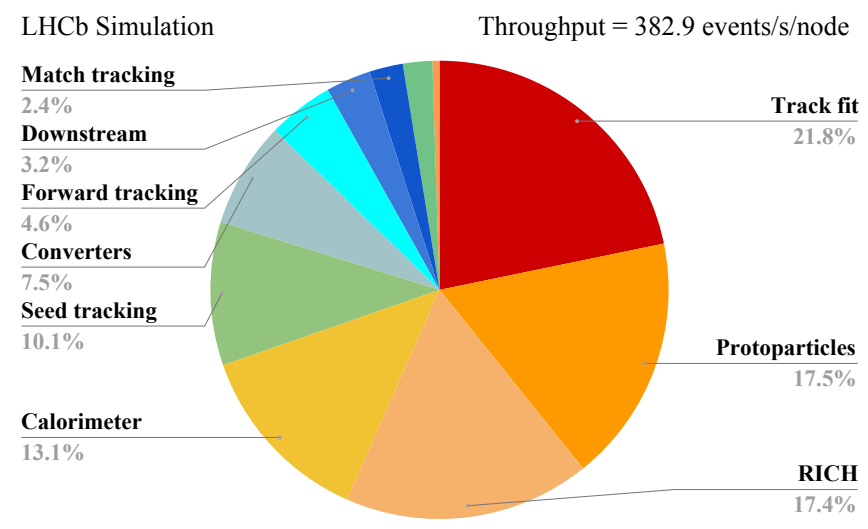

Figure 1: Breakdown of the current HLT2 reconstruction throughput rate for the LHCb upgrade using the "fastest" configuration [11].

\subsection{Tracking performance}

Two main types of tracks are used in LHCb analyses: long and downstream. Long tracks pass through the whole tracking system from the Vertex Locator (VELO), to the Upstream Tracker (UT) and Scintillating Fiber Tracker (SciFi) which are located upstream and downstream of the magnet, respectively. Downstream tracks usually come from the decay of long-lived particles (e.g. $K_{S}^{0}$ and $\Lambda^{0}$ ), and contain hits only in the UT and SciFi detectors.

A sum of typical simulation samples of $B_{s}^{0} \rightarrow J / \psi \phi, B_{s}^{0} \rightarrow \phi \phi, B^{0} \rightarrow K^{* 0} e^{+} e^{-}$and $D^{+} \rightarrow K_{s}^{0} \pi^{+}$signals is used to represent the main physics programme of LHCb and evaluate the tracking performance. Samples are generated with 5000 events per magnet polarity. The long and downstream tracks reconstruction efficiencies for particles originating from B or D decays after the track fitting are shown in Fig. 2. The corresponding fake rate and relative resolution of momentum of the reconstructed tracks are shown in Fig. 3. Excellent momentum resolution $(\delta p / p \sim 0.5 \%)$ is achieved in the whole momentum range.

\subsection{ECAL performance}

Information from the electromagnetic calorimeter (ECAL) is used in HLT2 to reconstruct neutral objects, such as photons and $\pi^{0}$, and as input to particle identification, with critical impact

\footnotetext{
${ }^{2}$ An optimized reconstruction sequence achieves higher throughput by removing the redundancy in the tracking reconstruction, by using a simplified version of the track fit and by exploiting a selective matching between tracks and calorimeter clusters.

3250 events/s/node is roughly estimated by assuming $1 \mathrm{MHz}$ input event rate with 2000 nodes multiplied by a factor of two taking into account the disk buffer.
} 

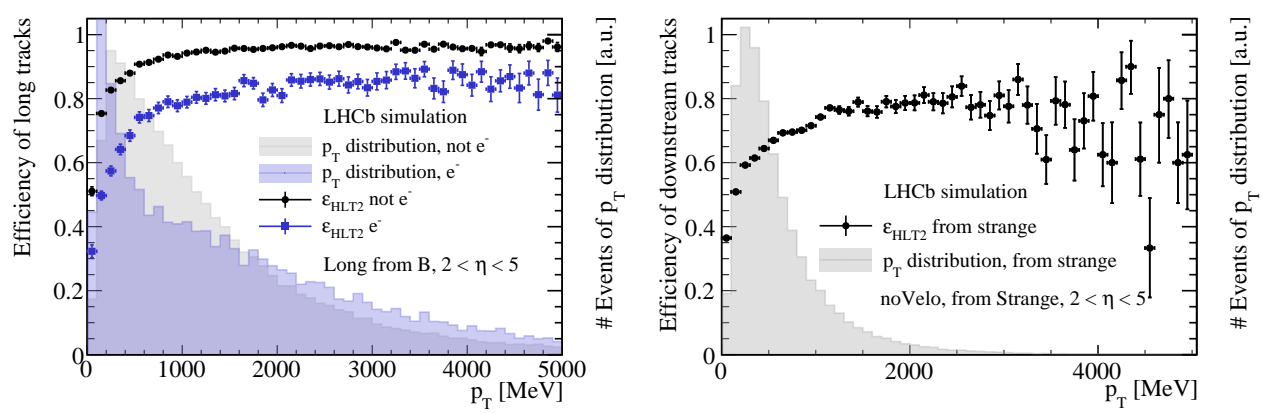

Figure 2: Long (left) and downstream (right) tracks reconstruction efficiencies versus transverse momentum $p_{T}$ for long and downstream reconstructible particles from $\mathrm{B}$ or $\mathrm{D}$ decays within $2<\eta<5$ [11]. The underlying histograms show the corresponding distributions of the reconstructible particles.
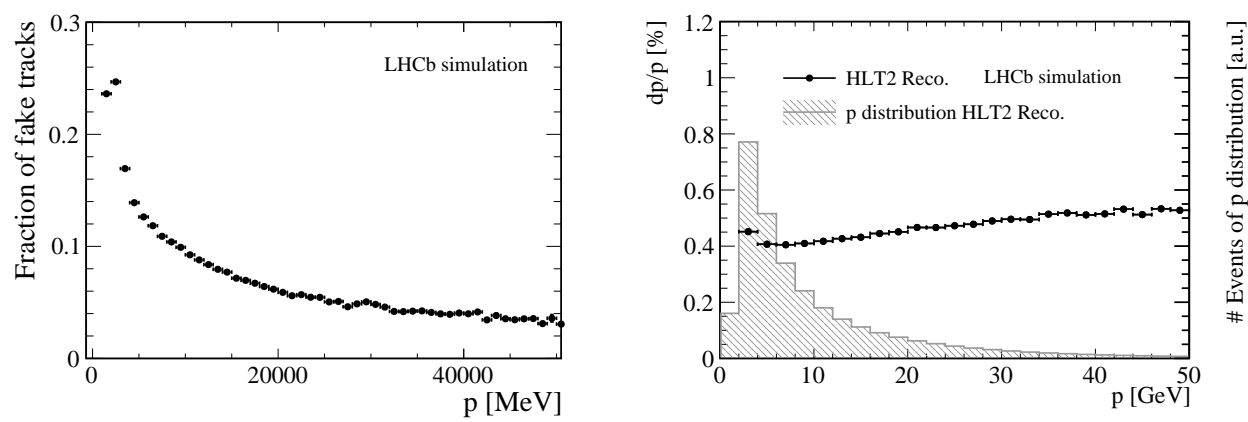

Figure 3: Left: Fake rate of the reconstructed long tracks as a function of transverse momentum $p_{T}$. Right: Relative resolution of the momentum of reconstructed tracks as a function of momentum $p$, where the underlying histogram shows the distribution of momentum. [11].

on electron identification. The efficiency of reconstructing ECAL clusters from the energy deposited by photons and the position resolution of the reconstructed clusters are estimated with a simulated sample of $B^{0} \rightarrow K^{* 0} \gamma$ decay and shown in Fig. 4. The different sizes of the ECAL cells in three ECAL regions result in different resolutions, in particular for the position resolution. The power of the ECAL variables to separate electrons from other charged particles is tested with a simulated sample of $B^{0} \rightarrow K^{* 0} e^{+} e^{-}$decay and shown in Fig. 5 for two key variables: the distribution of the ECAL energy over the track momentum, and the compatibility between the projection of the track direction before bending in the magnetic field and deposited energy in ECAL from potential bremsstrahlung emission. A good separation between electrons and charged hadrons is illustrated.

\section{Selective persistency}

Benefiting from the fully offline-quality reconstruction in HLT2, dedicated exclusive selections with ultimate precision are achieved in the real-time processing, where tight timing constraints required improvements in the selection algorithms. A new scheduling algorithm [13] for the upgrade software framework of $\mathrm{LHCb}$ was implemented to handle arbitrary data and control flow and supports multi-threading via inter-event concurrency for HLT2 selections. Moreover, particle selection algorithms were optimised to use a structure-of-array data format, which is better suited 

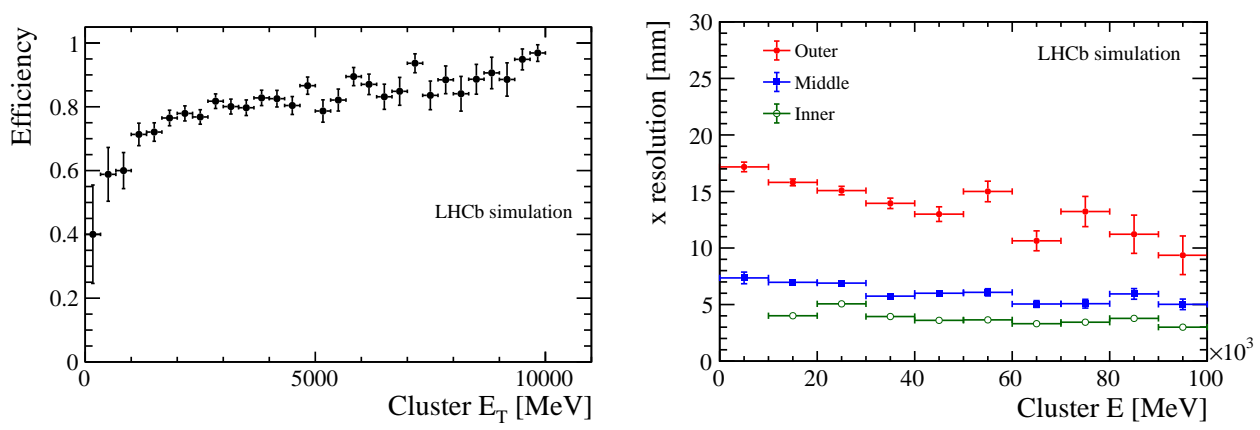

Figure 4: Left: ECAL cluster reconstruction efficiency versus transverse energy $E_{T}$ for reconstructible photons from B decays. Right: ECAL cluster $x$ position resolutions in three different regions versus energy for reconstructible photons from $B$ decays. [11].
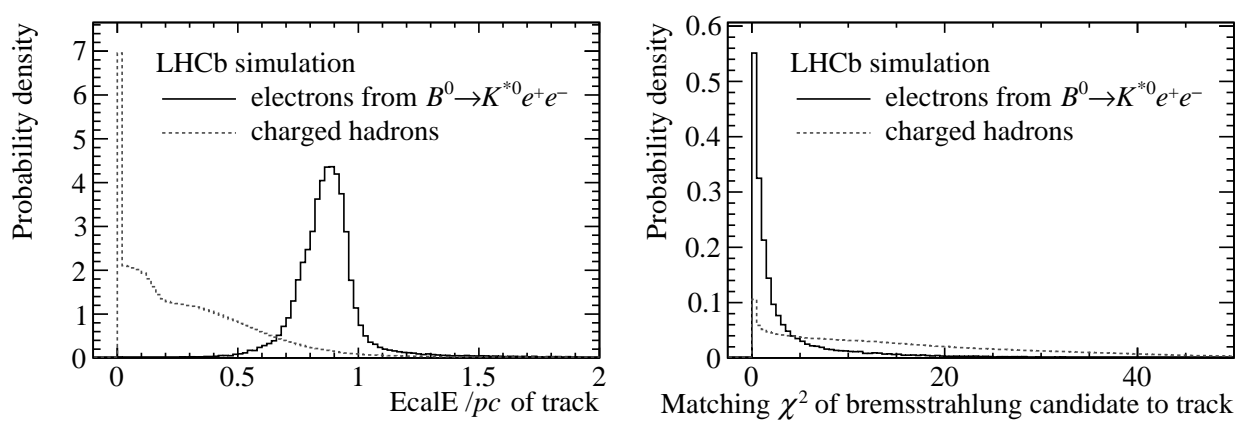

Figure 5: Left: Distribution of the cluster energy divied by the momentum of the matched tracks for signal (electrons) and background (charged hadrons). Right: $\chi^{2}$ of bremsstrahlung cluster candidates matching to tracks [11].

for optimisation using single instruction multiple data (SIMD) instructions supported by modern CPUs. As shown in Fig. 6, performance gains of up to 65\% are seen with the structure-of-arrays data model and AVX2 instruction set ${ }^{4}$, compared with the array-of-structures output data format used in Runs 1 and 2.

With an increase in the heavy flavour production rate, in addition to expected trigger efficiency gains and an increase in the raw event size, the trigger output bandwidth will increase by a factor of 15 from Run 2 [14]. Due to constraints on offline storage resources, the bandwidth is limited to a maximum of $10 \mathrm{~GB} / \mathrm{s}$ [14], for which it is impossible to save the full detector information for all $p p$ collisions containing decays of beauty and charm hadrons. To overcome this problem, the $\mathrm{LHCb}$ experiment has pioneered the usage of a reduced event format (a.k.a. Turbo stream) [16] since the beginning of Run 2. An improvement of the reduced event model has been developed to cope with beauty programmes which rely on inclusive triggers, where additional reconstructed objects in the event are selected and persisted after the trigger decision. As illustrated in Fig. 7, considering a trigger that reconstructs and selects the $D^{* \pm} \rightarrow D^{0}\left(\rightarrow K^{-} \pi^{+}\right) \pi^{ \pm}$decay, as a bare minimum only the objects involved in the trigger decision, plus the linked primary vertex (PV) are persisted in the

4The "Scalar", "SSE" and "AVX2" versions correspond to algorithms that use CPU instructions with registers holding 1, 4, and 8 32-bit floating-point numbers respectively. 


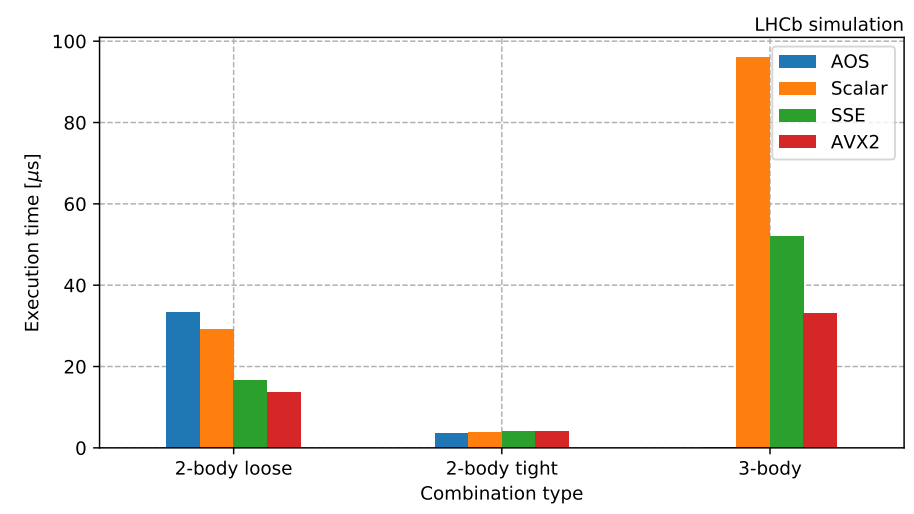

Figure 6: The timings of three different particle combination algorithms as performed by four different execution backends [15]. The execution times for the 2-body loose and 3-body algorithms are lower for backends which utilise vector instruction sets. The 2-body tight algorithm has a tight selection on its input objects and subsequently cannot fully fill the vector instruction registers during execution.

Turbo stream. The selective persistence (SP) allows for saving additional objects, e.g. other tracks coming from the same PV or tracks from other PVs, up to the full event, including possibly some raw data banks. This permits a significant event size reduction without sacrifice the possibility of performing exploratory analysis offline.

TURBO (since 2015)
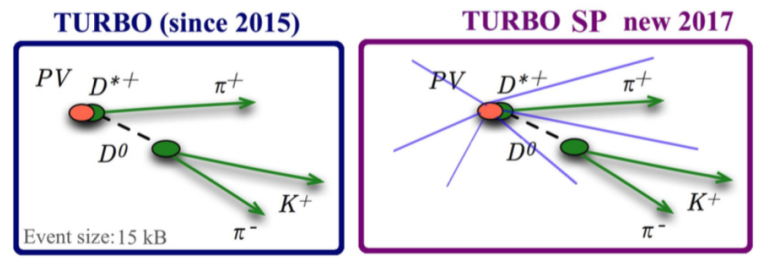

TURBO++ (since 2016)

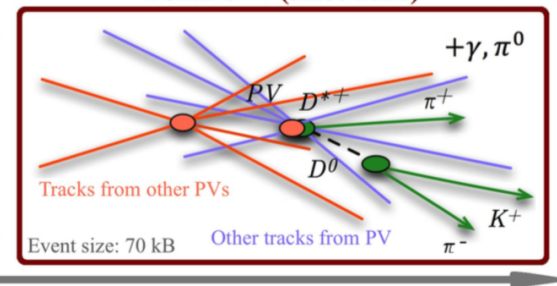

Event size

Figure 7: The Turbo model with different levels of object persistence [14]. Left to right illustrates the change of the event size by saving more additional information for a candidate event of $D^{*+} \rightarrow D^{0}\left(\rightarrow K^{-} \pi^{+}\right) \pi^{+}$ selected by HLT2.

\section{Summary}

The LHCb Collaboration is preparing a truly novel approach for the trigger system that will be operational in Run 3. The full detector will be read out for each beam crossing with $30 \mathrm{MHz}$ event rate and processed by a fully software trigger. The reconstruction, particle identification and event selections will be run online with offline quality thanks to quasi real-time calibration and alignment that will be performed on run-by-run basis. The throughput and physics performance are evaluated with simulated event samples and are shown to meet or exceed the performance goals defined in the trigger Technical Design Report [17].The offline-fidelity reconstruction and selective persistence will allow for a broader and explorable physics programme within limited computational resources for Run 3. 


\section{References}

[1] LHCb collaboration, Simultaneous determination of CKM angle $\gamma$ and charm mixing parameters, arXiv: 2110.02350.

[2] LHCb collaboration, Precise determination of the $B_{s}^{0}-\bar{B}_{s}^{0}$ oscillation frequency, arXiv: 2104.04221.

[3] LHCb collaboration, Analysis of neutral B-meson decays into two muons, arXiv: 2108.09284.

[4] LHCb collaboration, Observation of $J / \psi p$ resonances consistent with pentaquark states in $\Lambda_{b}^{0} \rightarrow J / \psi K^{-} p$ decays, Phys. Rev. Lett. 115, (2015) 072001.

[5] LHCb collaboration, Search for lepton-universality violation in $B^{+} \rightarrow K^{+} l^{+} l^{-}$decays, Phys. Rev. Lett. 122 (2019) 191801.

[6] S. Akar et al., Review Document: Low Level Trigger (LLT), LHCb-PUB-2014-037, CERN, Geneva, 2014.

[7] R. Aaij et al., Allen: A High-Level Trigger on GPUs for LHCb. Comput. Softw. Big Sci. 4, 7 (2020).

[8] Giulio Dujany et al., Real-time alignment and calibration of the LHCb Detector in Run II, 2015 J. Phys.: Conf. Ser. 664082010.

[9] LHCb collaboration, 2011 Letter of Intent for the LHCb Upgrade Tech. Rep. CERN-LHCC2011-001. LHCC-I-018.

[10] R. Aaij et al., A Comparison of CPU and GPU implementations for the LHCb Experiment Run 3 Trigger, LHCb-DP-2021-003.

[11] LHCb collaboration, Selected HLT2 reconstruction performance for the LHCb upgrade, LHCb-Figure-2021-003.

[12] C. Pérez and D. Hugo, LHCb Kalman Filter cross architecture studies, 2017 J. Phys.: Conf. Ser. 898032052.

[13] Ekaterina Govorkova et al., A new scheduling algorithm for the LHCb upgrade trigger application, LHCb-PROC-2020-003.

[14] LHCb collaboration, Computing Model of the Upgrade LHCb experiment, CERN-LHCC2018-014 (2018).

[15] LHCb collaboration, Comparison of particle selection algorithms for the LHCb Upgrade, LHCb-Figure-2020-018.

[16] R. Aaij et al., "A comprehensive real-time analysis model at the LHCb experiment", 2019 JINST 14 P04006.

[17] LHCb collaboration, LHCb Trigger and Online Upgrade Technical Design Report, CERNLHCC-2014-016. 